\title{
Anti-Inflammatory and Antioxidant Effects of Carpesium cernuum L. Methanolic Extract in LPS-Stimulated RAW 264.7 Macrophages
}

\author{
Yea-Jin Park, ${ }^{1}$ Se-Yun Cheon $\mathbb{D D}^{1,2}$ Dong-Sung Lee, ${ }^{3}$ Divina C. Cominguez, ${ }^{1}$ Zhiyun Zhang, \\ Sangwoo Lee, ${ }^{5}$ and Hyo-Jin An $\left(\mathbb{1}^{1}\right.$ \\ ${ }^{1}$ Department of Pharmacology, College of Korean Medicine, Sangji University, Wonju, Gangwon-do 26339, Republic of Korea \\ ${ }^{2}$ Department of Korean Medical Science, School of Korean Medicine and Healthy Aging Korean Medical Research Center, \\ Pusan National University, Yangsan, Gyeongnam, 50612, Republic of Korea \\ ${ }^{3}$ College of Pharmacy, Chosun University, 309 Pilmun-daero, Dong-gu Gwangju 61452, Republic of Korea \\ ${ }^{4}$ State Key Laboratory of Systematic and Evolutionary Botany, Institute of Botany, The Chinese Academy of Sciences, \\ Beijing 100093, China \\ ${ }^{5}$ International Biological Material Research Center, Korea Research Institute of Bioscience and Biotechnology, \\ Daejeon 34141, Republic of Korea
}

Correspondence should be addressed to Hyo-Jin An; sangjipharm@gmail.com

Received 7 February 2020; Revised 20 June 2020; Accepted 30 June 2020; Published 8 August 2020

Academic Editor: Michele T. Pritchard

Copyright (C) 2020 Yea-Jin Park et al. This is an open access article distributed under the Creative Commons Attribution License, which permits unrestricted use, distribution, and reproduction in any medium, provided the original work is properly cited.

\begin{abstract}
A hypernomic reaction or an abnormal inflammatory process could cause a series of diseases, such as cardiovascular disease, neurodegeneration, and cancer. Additionally, oxidative stress has been identified to induce severe tissue injury and inflammation. Carpesium cernuum L. (C. cernuum) is a Chinese folk medicine used for its anti-inflammatory, analgesic, and detoxifying properties. However, the underlying molecular mechanism of C. cernuum in inflammatory and oxidative stress conditions remains largely unknown. The aim of this study was to examine the effects of a methanolic extract of C. cernuum (CLME) on lipopolysaccharide- (LPS-) induced RAW 264.7 mouse macrophages and a sepsis mouse model. The data presented in this study indicated that CLME inhibited LPS-induced production of proinflammatory mediators such as nitric oxide (NO) and prostaglandin $\mathrm{E}_{2}\left(\mathrm{PGE}_{2}\right)$ in RAW 264.7 cells. CLME treatment also reduced reactive oxygen species (ROS) generation and enhanced the expression of heme oxygenase-1 (HO-1) protein in a dose-dependent manner in the LPS-stimulated RAW 264.7 cells. Moreover, CLME treatment abolished the nuclear translocation of nuclear factor $\kappa \mathrm{B}(\mathrm{NF}-\kappa \mathrm{B})$, enhanced the activation of nuclear factor-erythroid 2 p45-related factor 2 (Nrf2), and reduced the expression of extracellular signal-related kinase (ERK) and ERK kinase (MEK) phosphorylation in LPS-stimulated RAW 264.7 cells. These outcomes implied that CLME could be a potential antioxidant and anti-inflammatory agent.
\end{abstract}

\section{Introduction}

Inflammation is a multistep process occurring in many animals. It is also one of the first lines of defense against harmful stimuli, such as trauma, bacteria, and irritants [1]. Acute inflammation is a finite process, leading to the return of tissue homeostasis. However, it has been reported that unrestrained inflammation occurs during chronic inflammatory milieu, such as allergies, asthma, arthritis, atherosclerosis, multiple sclerosis, metabolic syndromes, and obesity $[2,3]$. Hence, controlling unbalanced inflammation is a potentially crucial strategy for preventing inflammatory environments. Macrophages detect and react to some pathogens through pattern-recognition receptors (PRRs) containing toll-like receptors (TLRs) and consequently regulate the inflammatory response [4]. Lipopolysaccharide (LPS), a well-known inflammatory ligand, can activate macrophages to release a variety of inflammatory cytokines [5]. Therefore, we can 
mimic inflammatory and oxidative stress milieu using RAW 264.7 macrophage cells, which demonstrate a highly reproducible response to LPS.

LPS stimulates TLR4 [6], and the LPS-initiated signaling cascade results in the activation of mitogen-activating protein kinase (MAPK) and the nuclear factor $-\kappa \mathrm{B}(\mathrm{NF}-\kappa \mathrm{B})$ inflammatory signaling pathway [7]. Moreover, activated macrophages result in the secretion of many proinflammatory molecules including reactive oxygen species (ROS), nitric oxide (NO), inducible nitric oxide synthase (iNOS), and prostaglandin $\mathrm{E}_{2}\left(\mathrm{PGE}_{2}\right)$ that have been known to be important components in tissue destruction and the associated pathological process $[8,9]$.

Oxidative stress is an important inducer for inflammation [10]. High levels of ROS can induce a considerable decrease in the antioxidant defense mechanisms resulting in DNA, protein, and lipid damage [11]. Furthermore, ROS modulate the intracellular reduction-oxidation- (redox-) sensitive signaling and nuclear transcription factors including NF- $\kappa \mathrm{B}$ and nuclear factor-erythroid 2 p 45 -related factor 2 (Nrf2) in LPS-challenged macrophage activation [12]. Nrf2 signaling constitutes one of the most prominent cellular defense mechanisms in xenobiotic damage and oxidative stress [13] and is ubiquitously expressed in extensive cell types and tissues [10]. In addition, Nrf2 is a chief transcription factor regulating heme oxygenase-1 (HO-1) and displays its effects by translocating to the nucleus [14]. HO-1 is an antioxidant enzyme induced by oxidative stress; however, overexpression of $\mathrm{HO}-1$ prior to stimulation with LPS remarkably declined the production of subsequent inflammatory mediators such as NO, interleukin-6 (IL-6), and monocyte chemoattractant protein-1 (MCP-1) [15]. In this respect, antioxidants are the first line of defense against oxidative damage induced by scavenging radicals and thus act to maintain optimal health [16].

Carpesium cernuum L. (C. cernuum), drooping carpesium, is a member of Compositae, and this is a Chinese folk medicine used for eliminating heat and toxic material, detumescence, and relieving pain [17]. More precisely, C. cernuum has been used to treat tonsillitis, mumps, dysentery, toothache, and mastitis in China [18]. Furthermore, some compounds isolated from C. cernuum were reported to possess antiplasmodial activity against Plasmodium falciparum and Plasmodium berghei in mice [19]. However, no report on the underlying molecular mechanism of C. cernuum in inflammation and oxidative stress has been recorded. Accordingly, this current study was designed to investigate the effects of methanolic extract of C. cernuum (CLME) on LPS-induced inflammation and oxidative stress via targeting the expression of $\mathrm{NF}-\kappa \mathrm{B}, \mathrm{Nrf} 2 /$ Kelch-like $\mathrm{ECH}$-associated protein 1 (Keap1), and extracellular signal-regulated kinase (ERK) in RAW 264.7 macrophages. Furthermore, we also examined the impact of CLME on an LPS-induced sepsis mouse model.

\section{Materials and Methods}

2.1. Chemicals and Reagents. CLME were obtained from the International Biological Material Research Center (Daejeon,
Republic of Korea). Dulbecco's modified Eagle's medium (DMEM), fetal bovine serum (FBS), penicillin, and streptomycin were purchased from Life Technologies Inc. (Grand Island, NY, USA). LPS (Escherichia coli, serotype 0111:B4), 3-(4,5-dimethylthiazol-2-yl)-2,5-diphenyltetrazolium bromide (MTT), $\mathrm{N}^{6}$-(1-Iminoethyl)-L-lysine (NIL), NS-398, Griess reagent, and (1R)-(-)-Myrtenal ( $\geq 95 \%)$ were purchased from Sigma-Aldrich (St. Louis, MO, USA). Dimethyl sulfoxide (DMSO) was purchased from Junsei Chemical Co., Ltd. (Tokyo, Japan). The inhibitor of $\kappa \mathrm{B}(\mathrm{I} \kappa \mathrm{B})$ (cat. No. sc203), phospho- (p-) I $\kappa$ B (cat. No. sc-8404), p65 (cat. No. sc109), Keap1 (cat. No. sc-514914), HO-1 (cat. No. sc-136960), ERK kinase (MEK) (cat. No. sc-81504), p-MEK (cat. No. sc81503), $\alpha$-tubulin (cat. No. sc-8035), and $\beta$-actin (cat. No. sc-47778) monoclonal antibodies, as well as the peroxidaseconjugated secondary antibody, were purchased from Santa Cruz Biotechnology, Inc. (Santa Cruz, CA, USA). iNOS (cat. No. \#2982), Nrf2 (cat. No. \#12721), ERK 1/2 (cat. No. \#9102), p-ERK 1/2 (cat. No. \#9101), p38 (cat. No. \#9212), p-38 (cat. No. \#9211), c-Jun N-terminal kinase (JNK) (cat. No. \#9252), p-JNK (cat. No. \#9251), and poly-adenosine diphosphate (ADP) ribose polymerase (PARP) (cat. No. \#9542) antibodies were purchased from Cell Signaling Technology (Danbers, MA, USA). The enzyme immunoassay kits for $\mathrm{PGE}_{2}$, tumor necrosis factor- $\alpha$ (TNF- $\alpha$ ), and IL-6 were obtained from R\&D Systems (Minneapolis, MN, USA). MUSE $^{\circledR}$ oxidative stress assay kit for ROS was obtained from Merck (Darmstadt, Germany).

2.2. Preparation of CLME. CLME was obtained from the International Biological Material Research Center (Daejeon, Republic of Korea). More precisely, C. cernuum (8BF2C2689F3D-4496-ADD8-9F3ABCBC5590) was collected from the Zheshang Park, Wuhu City, Anhui Province, in China and was identified by Dr. Zhiyun Zhang at the Herbarium, Institute of Botany, The Chinese Academy of Sciences in 2012. A voucher specimen (accession number KRIB 0050072) of the retained material is preserved at the herbarium of KRIBB. The dried and refined whole plant including roots of C. cernuum (32 g) was extracted with $1 \mathrm{~L}$ of $99.9 \%(v / v)$ methanol, with repeated sonication $(15 \mathrm{~min})$ and resting $(2 \mathrm{~h})$ for 3 days at $45^{\circ} \mathrm{C}$. The resultant product was filtered with nonfluorescence cottons and concentrated using the rotary evaporator (N-1000SWD, EYELA) under reduced pressure at $45^{\circ} \mathrm{C}$. Finally, a total of $3.52 \mathrm{~g}$ of CLME was obtained by freeze-drying and the yield was calculated at $11 \%$.

2.3. Animal Experiments of Septic Shock Model. Six-week-old male C57BL/6N mice were purchased from Daehan Biolink Co. (Daejeon, Republic of Korea). Prior to these experiments, the Institutional Animal Care and Use Committee (IACUC) of Sangji University approved all the experimental protocols (approved number 2019-18). Mice were maintained at $22 \pm$ $2^{\circ} \mathrm{C}$ and $55 \pm 9 \%$ humidity, under a $12 \mathrm{~h}$ light/dark cycle, and provided water and diet ad libitum. Mice were divided into three groups ( $n=5$ per group) as follows: LPS-treated group (LPS) and LPS+oral administration of CLME groups (CLME 50 or $100 \mathrm{mg} / \mathrm{kg}$ ). LPS was dissolved in phosphatebuffered saline (PBS), and CLME was dissolved in 18:1:1 
ratio of distilled water, ethanol, and cremophor (vehicle). The mice were orally administered either vehicle alone or vehicle with CLME for $1 \mathrm{~h}$ before LPS injection $(25 \mathrm{mg} / \mathrm{kg})$. The survival was recorded at different intervals.

2.4. Cell Culture. The RAW 264.7 cell line was obtained from the Korea Cell Line Bank (KCLB, Seoul, Republic of Korea). The cells were cultured in DMEM supplemented with $10 \%$ FBS, penicillin $(100 \mathrm{U} / \mathrm{mL})$, and streptomycin $(100 \mu \mathrm{g} / \mathrm{mL})$ at $37^{\circ} \mathrm{C}$ and a humidified atmosphere of $5 \% \mathrm{CO}_{2}$. The CLME was dissolved in DMSO and filtered using Acrodisc $^{\circledR}$ Syringe Filters $0.2 \mu \mathrm{m}$ Supor ${ }^{\circledR}$ Membrane (Rall Life Sciences, MI, USA).

2.5. MTT Assay for Cell Viability. Cell viability was assessed using the MTT assay. Briefly, RAW 264.7 cells were seeded into a 96-well plate at a density of $1 \times 10^{4}$ cells per well and then treated with various concentrations of CLME for $24 \mathrm{~h}$. After treatment, CLME-treated cells were incubated with the MTT solution $(5 \mathrm{mg} / \mathrm{mL})$ for $4 \mathrm{~h}$ at $37^{\circ} \mathrm{C}$. After discarding the supernatant, the formazan product that formed in the cell was dissolved in DMSO and measured at $570 \mathrm{~nm}$ using an Epoch microplate spectrometer (BioTek, Winooski, VT, USA).

2.6. NO Assay. NO content was analyzed indirectly by measuring the supernatants of cultured RAW 264.7 cells for nitrite using the Griess reagent (1\% sulfanilamide in 5\% phosphoric acid, $1 \% \alpha$-naphthylamide in $\mathrm{H}_{2} \mathrm{O}$ ). RAW 264.7 cells were seeded into a 24 -well plate at a density of $5 \times 10^{5}$ cells per well and then treated with various concentrations of CLME for $1 \mathrm{~h}$. NIL was used as a positive iNOS selective inhibitor. After preincubation with CLME and NIL, the cells were stimulated with LPS $(1 \mu \mathrm{g} / \mathrm{mL})$ for $48 \mathrm{~h}$. Cell culture media $(50 \mu \mathrm{L})$ was mixed with $50 \mu \mathrm{L}$ of Griess reagent in a 96-well plate, incubated at room temperature for $15 \mathrm{~min}$, and then measured at $540 \mathrm{~nm}$ using an Epoch microplate spectrometer (BioTek, Winooski, VT, USA).

2.7. $P G E_{2}$ Assay. RAW 264.7 cells were seeded into a 24 -well plate at a density of $5 \times 10^{5}$ cells per well and then treated with various concentrations of CLME for $1 \mathrm{~h}$. NS-398 was used as a positive cyclooxygenase-2 (COX-2) selective inhibitor. After preincubation with CLME and NS-398, the cells were stimulated with LPS $(1 \mu \mathrm{g} / \mathrm{mL})$ for $24 \mathrm{~h}$. The release of $\mathrm{PGE}_{2}$ in the cultured media was measured using the enzyme-linked immunosorbent assay (ELISA) kit according to the manufacturer's instructions.

2.8. ROS Assay. The cells were prepared in a $60 \mathrm{~mm}$ dish at a density of $1 \times 10^{6}$ cells and then treated with various concentrations of CLME for $1 \mathrm{~h}$. After preincubation with CLME, the cells were activated with LPS $(1 \mu \mathrm{g} / \mathrm{mL})$ for $1 \mathrm{~h}$ and mixed with Muse ${ }^{\circledR}$ Oxidative Stress Reagent working solution. The cells were incubated for $30 \mathrm{~min}$ at $37^{\circ} \mathrm{C}$ and detected using the Muse ${ }^{\circledR}$ Cell Analyzer (Merck, Darmstadt, Germany).

2.9. Preparation of the Nuclear and Cytosolic Extract. The cells were plated in $60 \mathrm{~mm}$ dishes $\left(1 \times 10^{6}\right.$ cells $\left./ \mathrm{mL}\right)$ and treated with CLME for $1 \mathrm{~h}$. And then, cells were stimulated with LPS $(1 \mu \mathrm{g} / \mathrm{mL})$ to check the expression of NF- $\kappa \mathrm{B}$ and
$\mathrm{Nrf} 2$ for $30 \mathrm{~min}$ and $1 \mathrm{~h}$, respectively. For cytosolic extraction, the hypotonic buffer (10 mM HEPES (pH 7.9), $1.5 \mathrm{mM}$ $\mathrm{MgCl}_{2}, 10 \mathrm{mM} \mathrm{KCl}, 0.2 \mathrm{mM}$ phenylmethylsulfonyl fluoride (PMSF), $0.5 \mathrm{mM}$ dithiothreitol (DTT), and $10 \mathrm{mg} / \mathrm{mL}$ aprotinin) was added to the cell. The cells are harvested by scraping with the hypotonic buffer and incubated on ice for $20 \mathrm{~min}$. The cells were then lysed by adding $0.1 \%$ Nonidet P- 40 , vortexed vigorously for $10 \mathrm{sec}$, and centrifuged at $12,000 \times \mathrm{g}$ for $5 \mathrm{~min}$. The supernatant was used for cytosolic extracts. For nuclear extraction, the pellet was resuspended in a high salt buffer (20 mM HEPES (pH 7.9), 25\% glycerol, $400 \mathrm{mM} \mathrm{KCl,}$ $1.5 \mathrm{mM} \mathrm{MgCl}_{2}, 0.2 \mathrm{mM}$ ethylenediaminetetraacetic acid (EDTA), $0.5 \mathrm{mM}$ DTT, $1 \mathrm{mM} \mathrm{NaF}$, and $1 \mathrm{mM}$ sodium orthovanadate), vortexed for $30 \mathrm{~min}$ at $4^{\circ} \mathrm{C}$, and centrifuged at $12,000 \times \mathrm{g}$ for $15 \mathrm{~min}$. The resulting supernatant was sued for nuclear extracts.

2.10. Western Blot Analysis. The cells were plated in $60 \mathrm{~mm}$ dishes $\left(2 \times 10^{5}\right.$ cells $\left./ \mathrm{mL}\right)$ and treated with CLME. After $1 \mathrm{~h}$, the LPS $(1 \mu \mathrm{g} / \mathrm{mL})$ was treated to the cells in order to examine the expression of p-MEK for $20 \mathrm{~min}, \mathrm{I} \kappa \mathrm{B}, \mathrm{p}-\mathrm{I} \kappa \mathrm{B}$, and MAPKs for $30 \mathrm{~min}$ and iNOS for $24 \mathrm{~h}$. First, cells were suspended in PRO-PREP ${ }^{\mathrm{TM}}$ protein extraction solution (Intron Biotechnology, Seoul, Republic of Korea). The suspension was incubated on ice for $20 \mathrm{~min}$ and then centrifuged at $11,000 \times \mathrm{g}$ for $30 \mathrm{~min}$. The protein concentration was determined using the Bio-Rad protein assay reagent according to the manufacturer's instructions (Bio-Rad, Hercules, CA, USA). Equal amounts $(20 \mu \mathrm{g})$ of the protein samples were separated on a sodium dodecyl sulfate (SDS) polyacrylamide gel, followed by transfer onto a polyvinylidene fluoride (PVDF) membrane. Membranes were incubated for $30 \mathrm{~min}$ with $2.5 \%$ skim milk at $20-25^{\circ} \mathrm{C}$ and then incubated overnight with a $1: 1000$ dilution of the primary antibody at $4^{\circ} \mathrm{C}$. The blots were washed three times with Tween 20/Tris-buffered saline (T/TBS) and incubated with a $1: 2500$ dilution of horseradish peroxidase-conjugated secondary antibody for $2 \mathrm{~h}$ at 20 $25^{\circ} \mathrm{C}$. The blots were further washed three times with T/TBS and finally visualized using enhanced chemiluminescence (GE Healthcare, Waukesha, WI, USA).

2.11. HPLC Analysis. HPLC was performed to identify the component profile of CLME. The extract was precisely quantified ( $5 \mathrm{mg}$ ), dissolved in $1 \mathrm{~mL}$ of $\mathrm{MeOH}$, and filtered to prepare an extract sample. The HPLC and ODS-A HPLC column (YMC-pack ODS-A, $250 \times 4.6 \mathrm{~mm}$ I.D., S-5 $\mu \mathrm{m}$, $12 \mathrm{~nm}$, YMC, Kyoto, Japan), consisting of a pump (1525 Binary HPLC pump, Waters, MA, USA) and a PDA detector (996 Photodiode array detector, waters, MA, USA), were connected for analysis. As mobile solvent systems, A channel: $0.1 \%$ acetic acid distilled water, and B channel: $0.1 \%$ acetic acid acetonitrile, were used. The slope profile proceeded as follows: $0-5 \mathrm{~min}, 5-10 \%$ B linear; $5-15 \mathrm{~min}, 10 \%$ B linear; $15-20 \mathrm{~min}, 10-20 \%$ B linear; $20-40 \mathrm{~min}, 20 \%$ B linear; 40 $60 \mathrm{~min}, 20-70 \% \mathrm{~B}$ linear; and $60-70 \mathrm{~min}, 70-100 \% \mathrm{~B}$. The flow rate was maintained at $1.0 \mathrm{~mL} / \mathrm{min}$ during the analysis. The CLME solution $(20 \mu \mathrm{L})$ was injected at $5 \mathrm{mg} / \mathrm{mL}$, and $20 \mu \mathrm{L}$ of $25 \mu \mathrm{g} / \mathrm{mL}$ of (1R)-(-)-Myrtenal ( $\geq 95 \%$, Sigma-Aldrich, St. Louis, MO, USA) dissolved in $\mathrm{MeOH}$ was injected as a 
standard sample. The detection wavelength was adjusted to $252 \mathrm{~nm}$. The different concentrations of (1R)-(-)-Myrtenal $(1.5625,3.125,6.25,12.5,25$, and $50 \mu \mathrm{g} / \mathrm{mL})$ were subjected to quantitative analysis using the calibration graph.

2.12. Cytokine Assay. The cells were transferred into 24 -well dishes at a density of $1 \times 10^{5}$ cells and then treated with various concentrations of CLME for $1 \mathrm{~h}$. Next, the cells were activated with LPS $(1 \mu \mathrm{g} / \mathrm{mL})$ for $24 \mathrm{~h}$, and the culture media were collected and stored at $-80^{\circ} \mathrm{C}$. The levels of TNF- $\alpha$ and IL-6 were measured using ELISA kits according to the manufacturer's instructions.

2.13. Statistical Analysis. Each result is expressed as the mean \pm standard deviation (S.D.) of triplicate experiments. Statistical analysis was performed using SPSS statistical analysis software (version 19.0; International Business Machines, Armonk, NY, USA). Statistically significant differences were determined using analysis of variance and Dunnett's post hoc test, and $P$ values of less than 0.05 were considered statistically significant.

\section{Results}

3.1. Effect of CLME on Survival Rate in LPS-Induced Septic Shock Mouse Model and Production of $\mathrm{NO}$ and $\mathrm{PGE}_{2}$ and Expression of iNOS Protein in LPS-Stimulated RAW 264.7 Cells. To examine the anti-inflammatory effect of CLME in vivo, mice were pretreated with CLME (50 or $100 \mathrm{mg} / \mathrm{kg}$ ) for $1 \mathrm{~h}$ and then injected with LPS $(25 \mathrm{mg} / \mathrm{kg})$. The survival was monitored every $10-12 \mathrm{~h}$ for 3 days. In the absence of CLME, $60 \%$ mice died within $28 \mathrm{~h}$ of LPS injection, and totally, $80 \%$ mice died within $41 \mathrm{~h}$. On the other hand, only $60 \%$ of septic mice with CLME-treated groups were dead within $41 \mathrm{~h}$ of LPS injection, indicating that CLME improved the survival of mice suffering from lethal endotoxic shock (Figure 1(a)). Prior to the in vitro antioxidant and antiinflammatory evaluation, we initially examined the effect of CLME on cytotoxicity in RAW 264.7 cells at the indicated concentrations $(15.63,31.25,62.5,125,250$, and $500 \mu \mathrm{g} / \mathrm{mL})$ using the MTT assay. As shown in Fig. S1A, the cell viability was unaffected by CLME at concentrations up to $31.25 \mu \mathrm{g} / \mathrm{mL}$, whereas higher concentrations of CLME showed obvious cytotoxicity. Therefore, concentrations of CLME less than $31.25 \mu \mathrm{g} / \mathrm{mL}(6.25,12.5$, and $25 \mu \mathrm{g} / \mathrm{mL})$ were chosen for the subsequent experiments. Furthermore, RAW 264.7 cell morphology was also observed under an optical microscope. The protective effect of CLME was revealed in the morphological changes induced by LPS stimulation. In cells pretreated with CLME, the level of cell spreading and pseudopodia induced by LPS was decreased, indicating inhibition of cell activation or differentiation (Fig. S1B). Redundant $\mathrm{NO}$ and $\mathrm{PGE}_{2}$, as inflammatory mediators, have been regarded as the main causes for acute inflammatory responses as well as chronic inflammatory diseases, such as osteoarthritis, rheumatoid arthritis, and inflammatory bowel disease [20]. In order to examine the effect of CLME on level of inflammatory mediators, we investigated $\mathrm{NO}$ and $\mathrm{PGE}_{2}$ production in LPS-stimulated RAW 264.7 cells. The cells were treated with or without CLME for $1 \mathrm{~h}$ and then treated with LPS $(1 \mu \mathrm{g} / \mathrm{mL})$. CLME treatment significantly $(P<0.001)$ suppressed LPS-induced NO production in a dose-dependent manner. In particular, high concentration of CLME $(25 \mu \mathrm{g} / \mathrm{mL})$ demonstrated elevated inhibitory effects on NO production than NIL, used as a positive iNOS selective inhibitor (Figure 1(b)). Moreover, LPS-induced $\mathrm{PGE}_{2}$ production was significantly $(P<0.001)$ decreased by CLME treatment in a dose-dependent manner, and NS-398 was also used as a positive COX-2 selective inhibitor (Figure 1(c)). The expression of the iNOS protein was increased after stimulation with LPS in RAW 264.7 cells, and we determined whether CLME could repress iNOS protein expression. As demonstrated by western blot analysis, cells pretreated with CLME strongly $(P<0.001)$ repressed the expression of the iNOS protein in a dose-dependent manner (Figure 1(d)). As a result, it can be established that CLME possesses anti-inflammatory ability through the mitigation of the proinflammatory enzyme, iNOS, and the mediators, $\mathrm{NO}$ and $\mathrm{PGE}_{2}$.

3.2. Effect of CLME on NF- $\kappa B$ Activation in LPS-Stimulated RAW 264.7 Cells. To establish the cellular mechanism involved in the anti-inflammatory activity of CLME-related inhibition of $\mathrm{NO}, \mathrm{PGE}_{2}$, and iNOS levels, the protein expressions of $\mathrm{I} \kappa \mathrm{B}, \mathrm{p}-\mathrm{I} \kappa \mathrm{B}$, and NF- $\kappa \mathrm{B}$ were detected in the LPSstimulated RAW 264.7 cells. As shown in Figure 2(a), a difference in $\mathrm{I} \kappa \mathrm{B}-\alpha$ expression was noted between the CON and LPS-treated groups. The CLME treatment $(25 \mu \mathrm{g} / \mathrm{mL})$ had notably higher I $\kappa \mathrm{B}$ expression than the LPS-treated group. Moreover, LPS treatment remarkably increased the phosphorylation of $\mathrm{I} \kappa \mathrm{B}$ compared to the $\mathrm{CON}$ group. Conversely, high concentration of CLME reported markedly $(P<0.001)$ lower phosphorylation of I $\kappa \mathrm{B}$ compared to the LPS-treated group. There was also a significant difference in p65 expression between the CON group and the LPS group, and the CLME-treated groups demonstrated significantly lower nuclear p65 expression than the LPStreated group $(P<0.001)$. Additionally, the CLME-treated group $(25 \mu \mathrm{g} / \mathrm{mL})$ showed notably $(P<0.001)$ higher cytosolic p65 expression than the LPS-treated group. Overall, the nuclear translocation of $\mathrm{NF}-\kappa \mathrm{B}$ was inhibited by CLME in a dose-dependent manner (Figure 2(b)). Notably, the suppression of NF- $\kappa \mathrm{B}$ protein exhibited a similar pattern to the inhibition of $\mathrm{NO}, \mathrm{PGE}_{2}$, and iNOS levels.

3.3. Effect of CLME on ROS Generation in LPS-Stimulated RAW 264.7 Cells. The elevated levels of ROS through modulation of signaling pathways boosts the generation of a proinflammatory condition and sustain disease progression [21]. To identify whether CLME has direct antioxidant potential in LPS-stimulated RAW 264.7 cells, the cellular ROS scavenging activity was assessed using the MUSE $^{\circledR}$ oxidative stress assay. ROS neg. (negative) and ROS pos. (positive) cells indicate M1 and M2, respectively. As shown in Figure 3, the LPS-treated group provoked a remarkable increase (28.95\%) in intracellular ROS generation compared to the CON group $(10.38 \%)$, whereas the CLME-treated groups resulted in $22.20,22.67$, and $19.75 \%$ on the ROS generation ratio in 


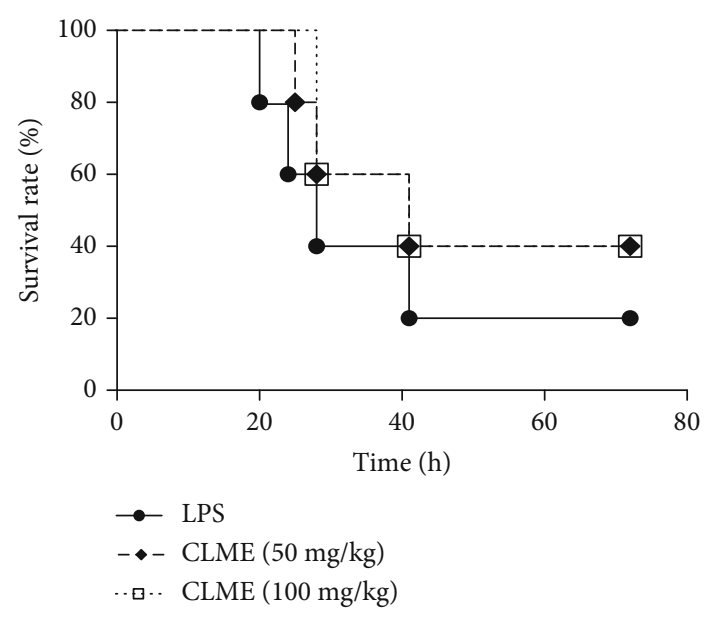

(a)

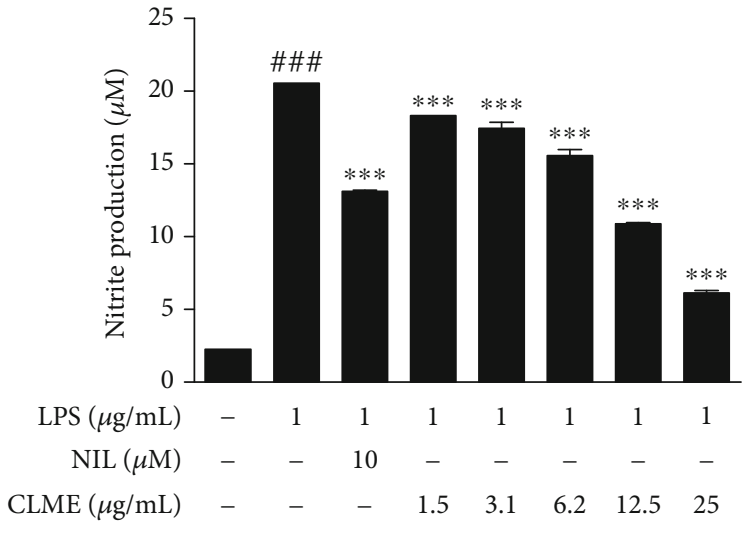

(b)
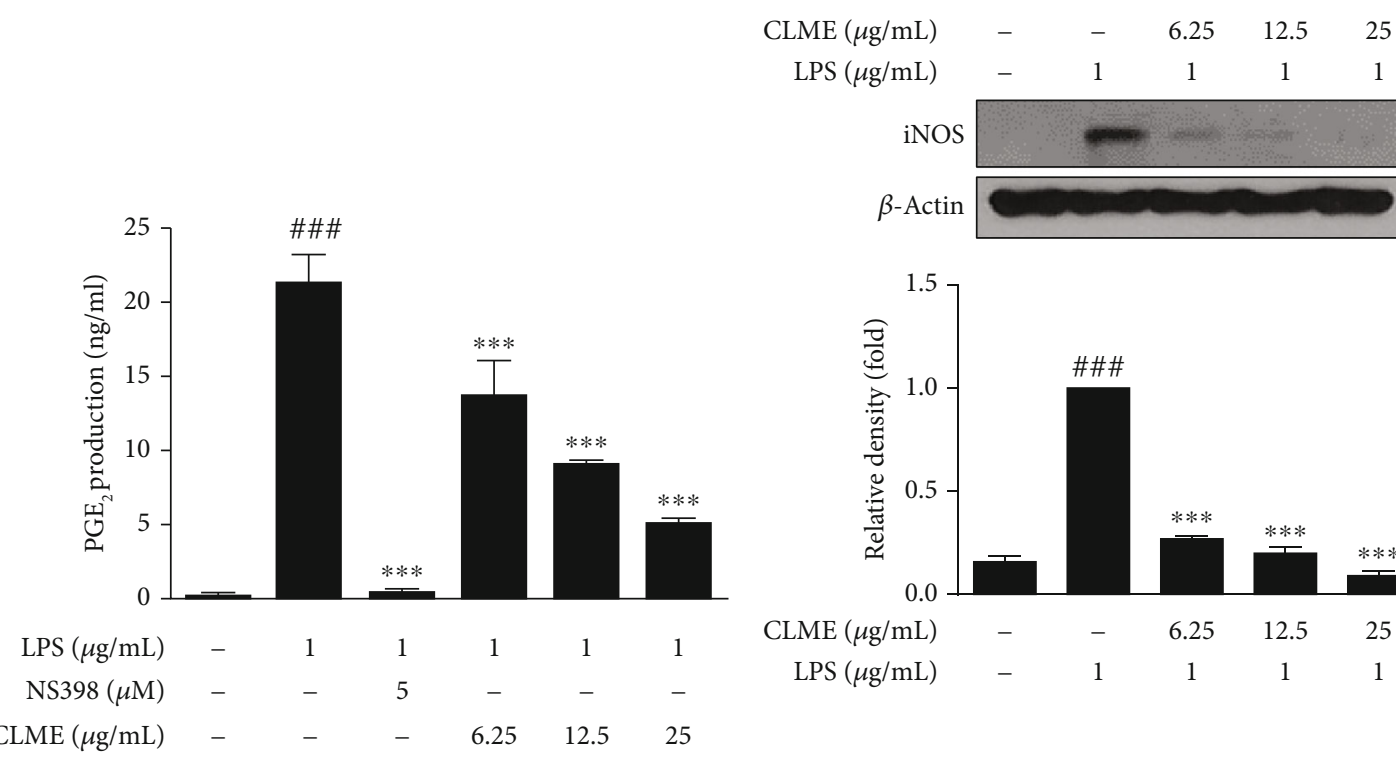

(c)

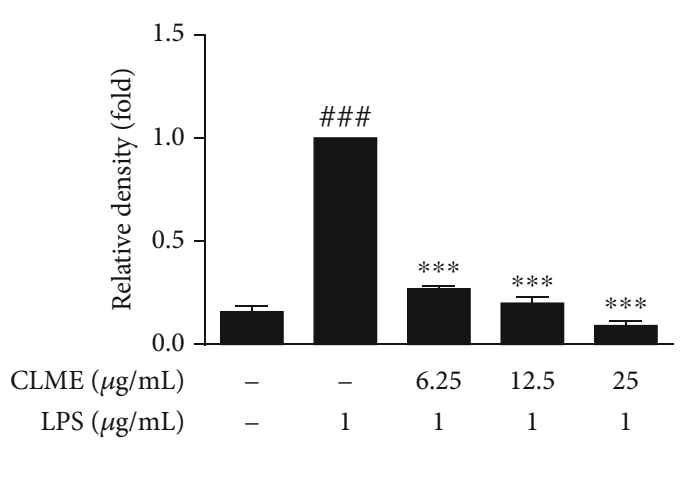

(d)

FIGURE 1: CLME prevents septic death in an LPS-induced endotoxemia mouse model and inhibits the levels of NO, PGE 2 , and iNOS in LPSstimulated RAW 264.7 cells. (a) The graph represents survival rate after LPS injection $(25 \mathrm{mg} / \mathrm{kg}$ ) with or without CLME treatment (50 or $100 \mathrm{mg} / \mathrm{kg}$ ). RAW 264.7 cells were pretreated with indicated concentrations of CLME for $1 \mathrm{~h}$ and followed by LPS stimulation ( $1 \mu \mathrm{g} / \mathrm{mL})$ for indicated times. (b) NO production was analyzed indirectly by measuring the supernatants of cultured RAW 264.7 cells for nitrite using the Griess reagent. (c) The prostaglandin $\mathrm{E}_{2}\left(\mathrm{PGE}_{2}\right)$ production in the culture media was measured by ELISA kit. (d) The iNOS expression was analyzed by western blot analysis. Densitometric analysis was performed using ImageJ ver. 1.50i. The values are represented as the mean \pm S.D. $(n=4) .{ }^{\# \# \#} P<0.001$ vs. the CON group; ${ }^{* * *} P<0.001$ vs. the LPS-treated group.

LPS-stimulated RAW 264.7 cells. Based on these findings, we demonstrated that CLME possesses antioxidant capacity by scavenging the generated ROS.

3.4. Effect of CLME on the Expression of HO-1 and Nrf2/Keap1 in LPS-Stimulated RAW 264.7 Cells. To clarify the impact of CLME on the expression of HO-1, a cellular antioxidant enzyme, RAW 264.7 cells were treated with LPS for $4,6,8,12$, and $24 \mathrm{~h}$. The expression of HO- 1 was increased by LPS treatment in RAW 264.7 cells, peaking at $12 \mathrm{~h}$ (Fig. S2A). In addition, the time-dependent western blot analysis indicated greatly $(P<0.001)$ increased protein expression of HO-1, from 6 to $12 \mathrm{~h}$, peaking at $12 \mathrm{~h}$ after CLME treatment (Figure 4(a)). Furthermore, the expression of HO-1 was also increased and maintained by CLME treatment during $12 \mathrm{~h}$ in LPS-stimulated RAW 264.7 cells (Fig. S2B). Based on the above results, we further confirmed the expression of $\mathrm{HO}-1$ at $12 \mathrm{~h}$ with three concentrations of CLME $(6.25,12.5$, and $25 \mu \mathrm{g} / \mathrm{mL})$. The data demonstrated that CLME gradually $(P<0.001)$ enhanced the expression of HO-1 in a dose-dependent manner in RAW 264.7 cells treated with or without LPS (Figures 4(b) and 4(c)). These results demonstrated that CLME possesses antioxidant activity by regulating ROS generation and HO-1 expression. Oxidative stressors and some anti-inflammatory electrophilic drugs interrupt Keap1-Nrf2 complex, promote the nuclear translocation of $\mathrm{Nrf} 2$, and finally activate the transcription of antioxidant genes including $\mathrm{HO}-1$ [22]. Therefore, we 

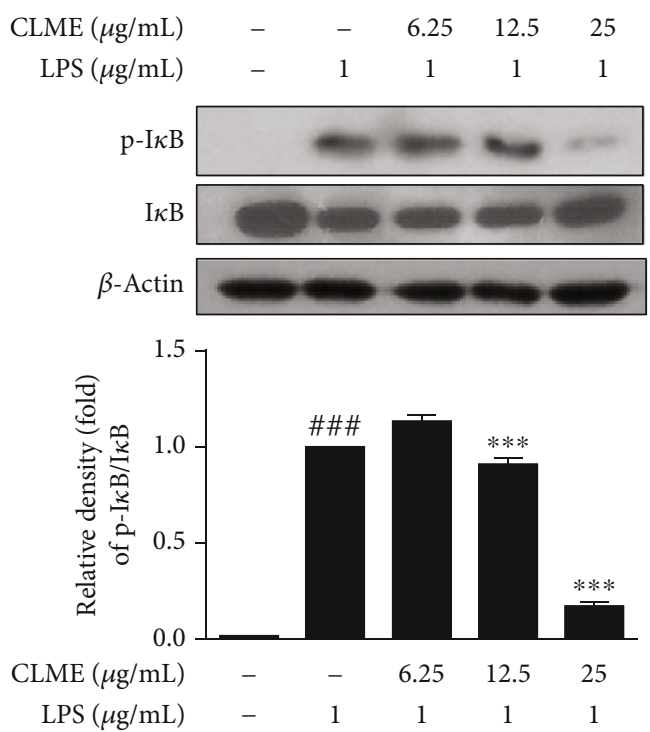

(a)
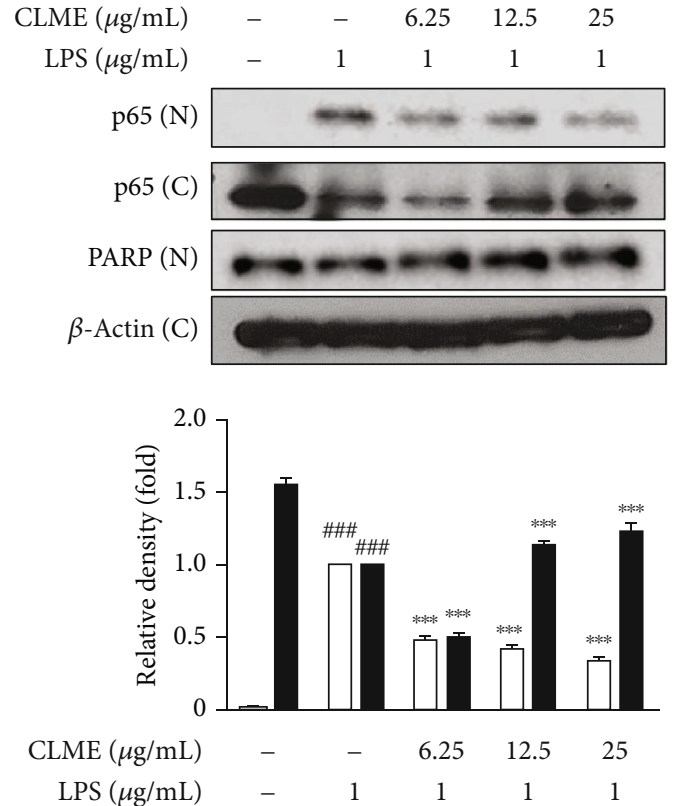

$\square \operatorname{pr} 65(\mathrm{~N})$

(b)

FIGURE 2: CLME inhibits the phosphorylation and degradation of I $\kappa$ B and nuclear translocation of NF- $\kappa$ B in LPS-stimulated RAW 264.7 cells. RAW 264.7 cells were pretreated with indicated concentrations of CLME for $1 \mathrm{~h}$ and followed by LPS stimulation $(1 \mu \mathrm{g} / \mathrm{mL})$ for $30 \mathrm{~min}$. (a) The $\mathrm{I} \kappa \mathrm{B}, \mathrm{p}-\mathrm{I} \kappa \mathrm{B}$, and (b) p65 expressions were analyzed by western blot analysis. Densitometric analysis was performed using Image $\mathrm{J}$ ver. $1.50 \mathrm{i}$. The values are represented as the mean \pm S.D. $(n=3) .{ }^{\# \#} P<0.001$ vs. the CON group; ${ }^{* * *} P<0.001$ vs. the LPS-treated group.

hypothesized that the regulatory effect of CLME on LPSinduced oxidative stress (e.g., intracellular ROS generation) and HO-1 expression may be controlled by the Keap1/Nrf2 signaling pathway. LPS treatment increased the cytosolic expression of the Keap1 protein. Conversely, CLME treatment dramatically $(P<0.001)$ attenuated LPS-induced cytosolic Keap1 expression (Figure 4(d)). Moreover, it was also noted that CLME treatment resulted in a strong $(P<0.001)$ upregulation in the nuclear accumulation of Nrf2 expression compared to the LPS group (Figure 4(e)). These results postulated that CLME activates the Keap1/Nrf2 signaling, shedding light on the molecular mechanism underlying its antioxidant effects.

3.5. Effect of CLME on MEK/ERK Activation in LPSStimulated RAW 264.7 Cells. To verify the hypothesis that CLME predominantly signals through the MAPK pathway in the context of NF- $\kappa \mathrm{B}$ inactivation and Nrf2 activation, we evaluated the effect of CLME on the MAPK signaling pathway in LPS-stimulated RAW 264.7 cells. As shown in Figure 5(a), the activation of MAPK (p38, ERK, and JNK) signaling occurred in the LPS-treated group compared to the CON group. CLME significantly $(P<0.001)$ declined the phosphorylation of ERK compared to the LPS group. However, the expressions of phosphorylated JNK and p38 MAPKs were unaffected by CLME. In addition, the impact of CLME on the expression of MEK, which is upstream of ERK, was evaluated, and CLME treatment effectively
$(P<0.001)$ alleviated the expression of $\mathrm{p}$-MEK compared to the LPS-treated group (Figure 5(b)).

3.6. Anti-Inflammatory Effect of Myrtenal as a Compound of CLME. We have confirmed the HPLC chromatogram of C. cernuum extract and the standard compound (1R)-(-)-Myrtenal. As shown in Figure 6(a), the retention time of the main peak in CLME was $60.629 \mathrm{~min}$. In addition, it was confirmed that the peak of (1R)-(-)-Myrtenal appeared at $60.629 \mathrm{~min}$ (Figure 6(b)), and CLME also showed a peak at the same retention time, $60.629 \mathrm{~min}$. Moreover, we also checked the content evaluation of (1R)-(-)-Myrtenal in the C. cernuum extract and the content values of (1R)-(-)-Myrtenal in the sample of CLME was $0.86 \pm 0.15 \mathrm{mg} / \mathrm{g}(0.086 \pm 0.015 \%)$. Therefore, this result suggested that CLME contained (1R)(-)-Myrtenal as one of the major compounds. Next, we examined the effect of Myrtenal on viability of RAW 264.7 cells, and $1000 \mu \mathrm{M}$ of Myrtenal demonstrated obvious cytotoxicity (Figure 6(d)). Therefore, three concentrations (125, 250, and $500 \mu \mathrm{M})$ of Myrtenal were chosen for the subsequent experiments. Similar to CLME, Myrtenal treatment $(500 \mu \mathrm{M})$ significantly $(P<0.001)$ suppressed LPS-induced production of NO, as well as the inflammatory cytokines TNF- $\alpha$ and IL-6, in a dose-dependent manner (Figures 6(e)-6(g)).

\section{Discussion}

Recent research has found various macrophages roles and functions, and macrophages play an important role in 


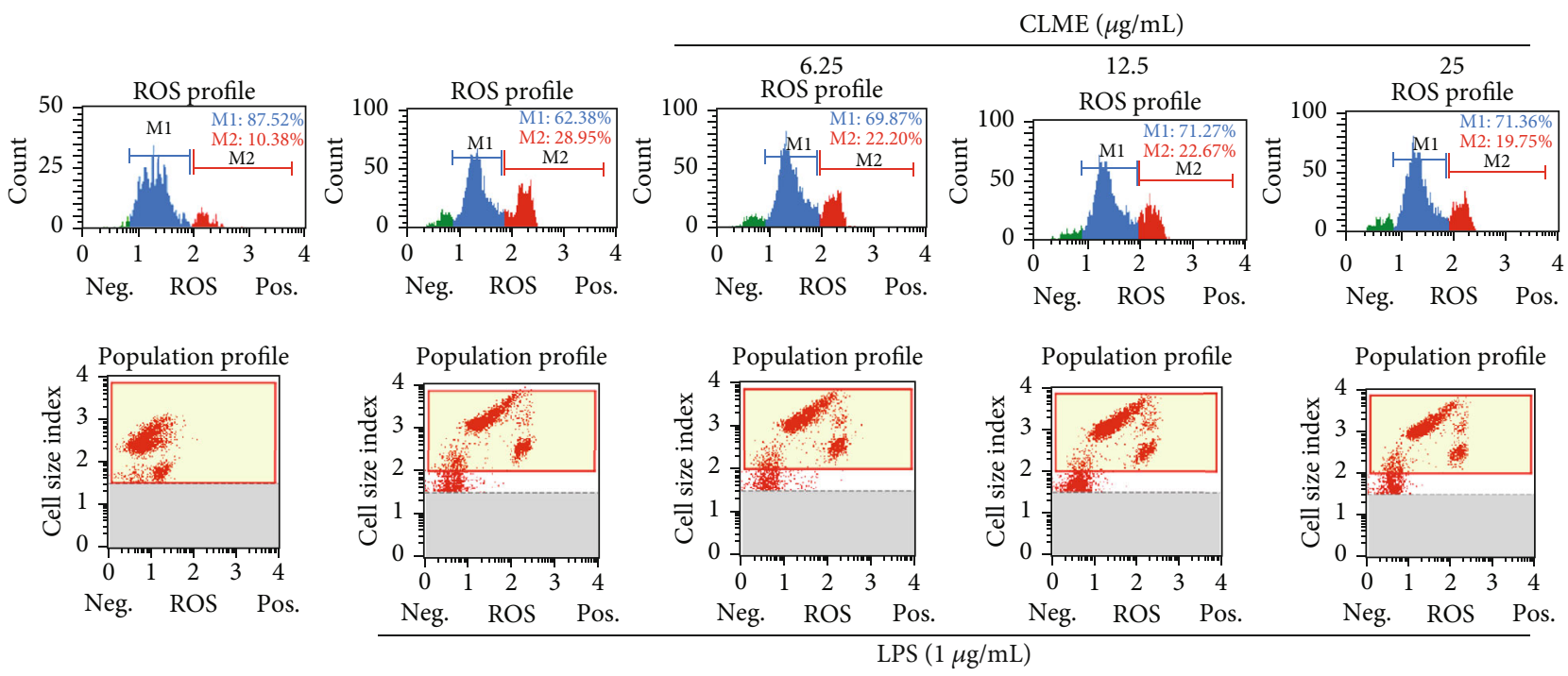

(a)

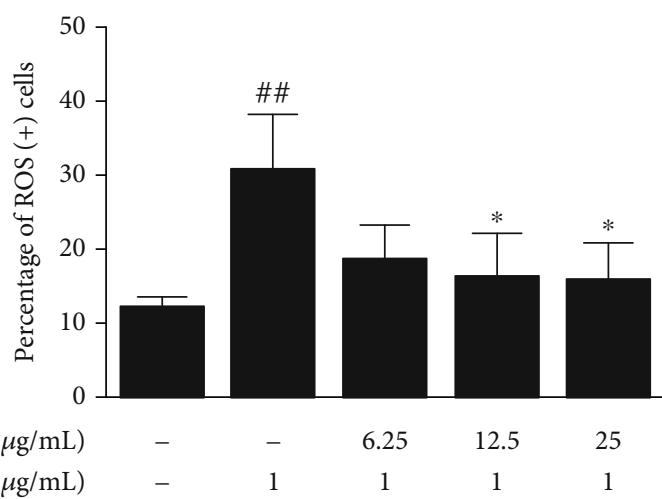

(b)

FIGURE 3: CLME inhibits the generation of ROS in LPS-stimulated RAW 264.7 cells. RAW 264.7 cells were pretreated with indicated concentrations of CLME for $1 \mathrm{~h}$ and followed by LPS stimulation $(1 \mu \mathrm{g} / \mathrm{mL})$ for $1 \mathrm{~h}$. (a) The plot shows the histogram of gated cells with two markers providing data on two cell populations: ROS neg. (-) and ROS pos. (+) cells, indicating M1 and M2, respectively. ROS generations were analyzed by MUSE $^{\circledR}$ oxidative stress assay kit. (b) The graph for percentage of ROS pos. $(+)$ cells. The values are represented as the mean \pm S.D. $(n=3) .{ }^{\# \#} P<0.01$ vs. the CON group; ${ }^{*} P<0.05$ vs. the LPS-treated group.

maintaining homeostasis and normal physiological conditions by coordinating a variety of biological activities [23]. Actually, studies examining the putative immunological properties of natural products and/or their derivatives depend heavily on the use of cell lines as an initial screen for biological activity [24]. In addition, because of their popularity as targeted single cells for assessing immune reactivity [25-28], we also chose RAW 264.7 macrophages for our current study. Macrophages can be stimulated by LPS to produce proinflammatory molecules (e.g., $\mathrm{NO}$ and $\mathrm{PGE}_{2}$ ) by activating intracellular signaling pathways, including NF- $\kappa \mathrm{B}$ and MAPK signaling pathways [29]. iNOS is the key enzyme responsible for the production of $\mathrm{NO}$ and is expressed excessively during an inflammatory response [8]. Accordingly, the production of these proinflammatory molecules is a major factor used to assess the efficacy of anti-inflammatory drugs.

NF- $\kappa \mathrm{B}$-activating pathways are triggered by a variety of extracellular stimuli, leading to the phosphorylation and subsequent proteasome-mediated degradation of inhibitory molecules, the inhibitor of NF- $\kappa \mathrm{B}(\mathrm{I} \kappa \mathrm{B})$ proteins [30], translocation of NF- $\kappa \mathrm{B}$ to the nucleus [31], and subsequent combination with their cognate DNA binding sites to modulate the transcription of more than 150 genes, such as proinflammatory cytokines, antimicrobial peptides, chemokines, antiapoptotic proteins, and stress-response proteins [32]. In this study, we noted that $\mathrm{I} \kappa \mathrm{B}$ degradation and phosphorylation and NF- $\kappa$ B translocation are effectively recovered by CLME in RAW 264.7 cells when triggered by LPS.

Chronic inflammation exerts its cellular side effects mainly through excessive production of free radicals and depletion of antioxidants [33]. Continued active inflammation response can lead to cell damage or cellular hyperplasia following ROS overproduction from inflammatory cells [33]. There is reversible interrelationship between oxidative stress and inflammation. The factors that cause inflammation and its amplification lead to oxidative stress and the reverse sequence of events (oxidative stress induced inflammation) [34]. ROS are the main oxidative products principally 


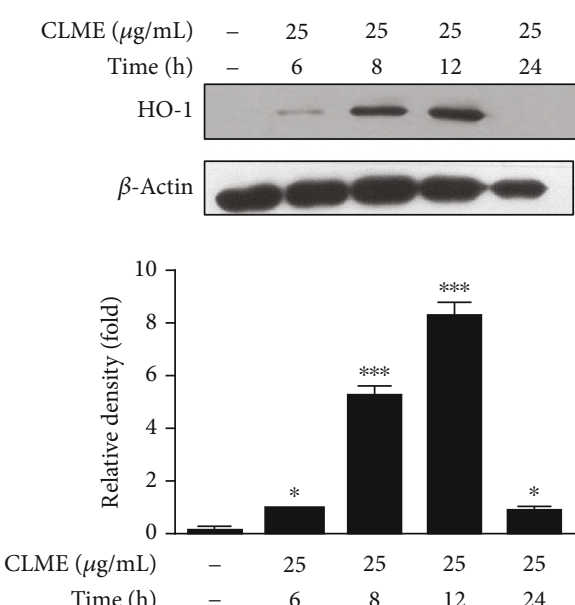

(a)
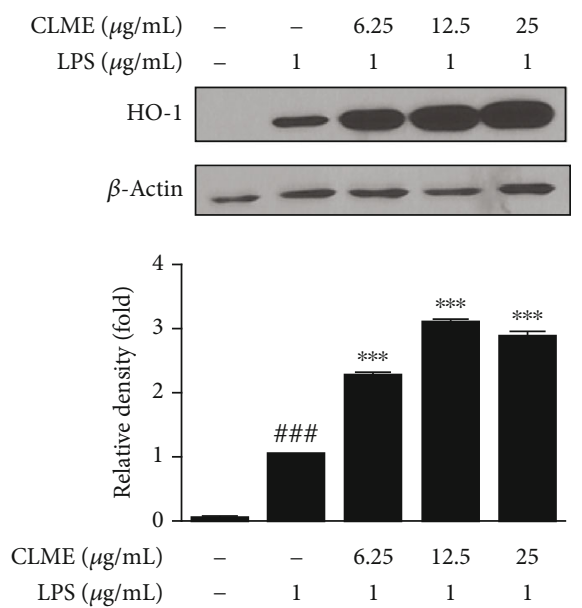

(c)
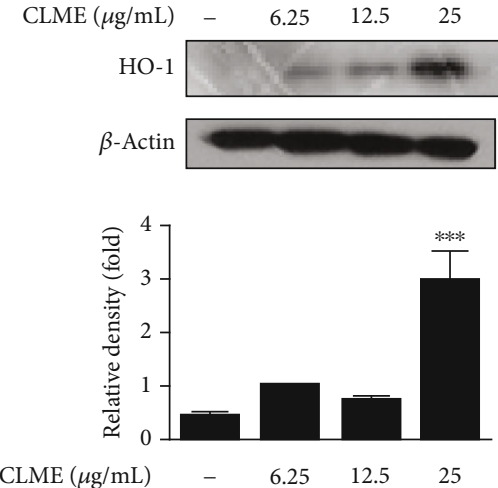

(b)
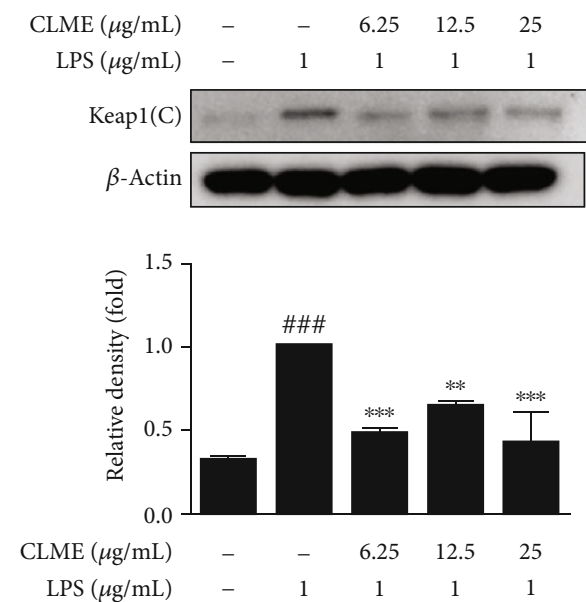

(d)

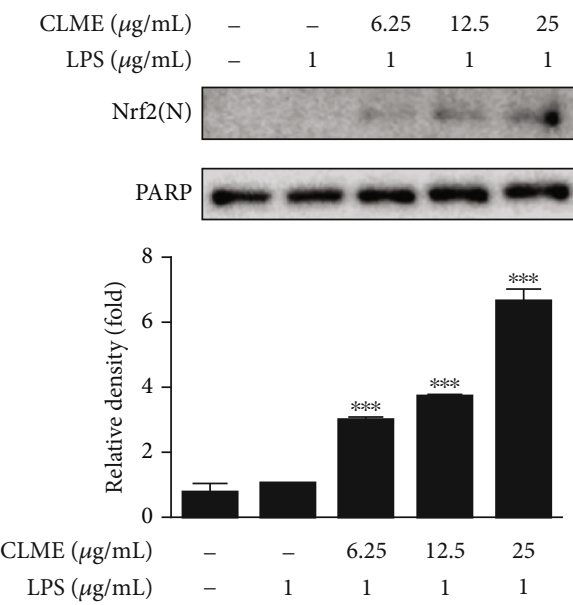

(e)

FIGURE 4: CLME regulates the expression of HO-1 and Keap1/Nrf2 in LPS-stimulated RAW 264.7 cells. (a-c) HO-1 expression in RAW 264.7 cells. (a) The cells were treated with CLME of $25 \mu \mathrm{g} / \mathrm{mL}$ for the indicated times. (b) The cells were treated with CLME of indicated concentrations for $12 \mathrm{~h}$. The values are represented as the mean \pm S.D. $(n=3)$. ${ }^{*} P<0.05$ and ${ }^{* * *} P<0.001$ vs. the CON group. (c) The cells were pretreated with indicated concentrations of CLME for $1 \mathrm{~h}$ and followed by LPS stimulation $(1 \mu \mathrm{g} / \mathrm{mL})$ for $12 \mathrm{~h}$. (d, e) The cells were pretreated with indicated concentrations of CLME for $1 \mathrm{~h}$ and followed by LPS stimulation for $1 \mathrm{~h}$. (d) Keap1 and (e) Nrf2 expressions were analyzed by western blot analysis. Densitometric analysis was performed using ImageJ ver. 1.50i. The values are represented as the mean \pm S.D. $(n=3)$. ${ }^{\# \#} P<0.001$ vs. the CON group; ${ }^{* *} P<0.01$ and ${ }^{* * *} P<0.001$ vs. the LPS-treated group. 


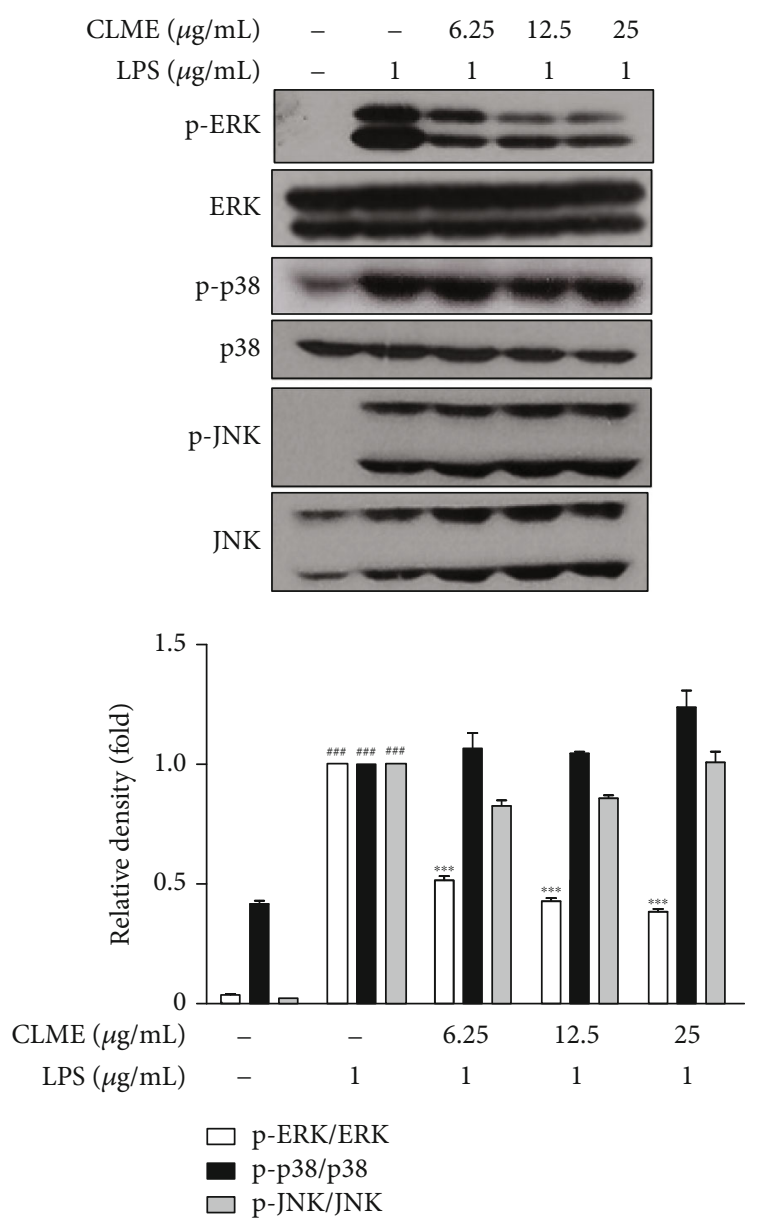

(a)
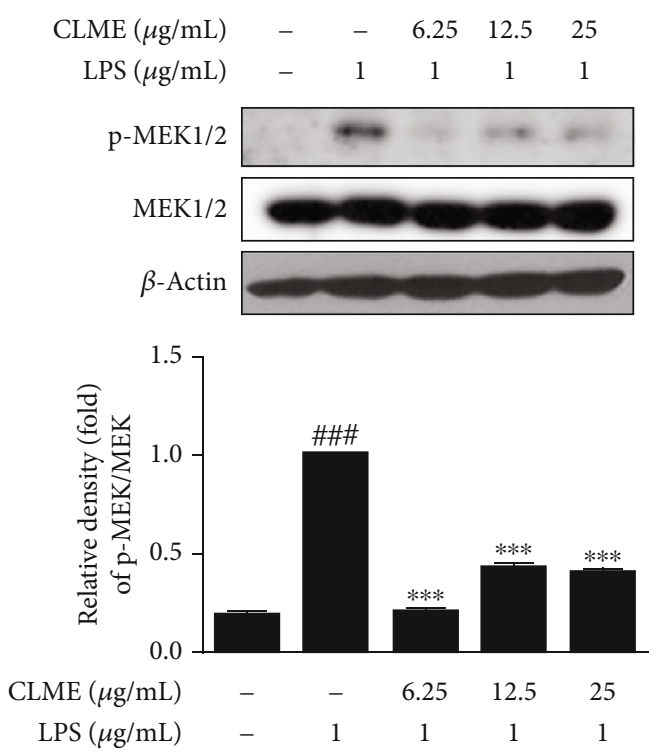

(b)

FIGURE 5: CLME inhibits the phosphorylation of MEK/ERK in LPS-stimulated RAW 264.7 cells. RAW 264.7 cells were pretreated with indicated concentrations of CLME for $1 \mathrm{~h}$ and followed by LPS stimulation ( $1 \mu \mathrm{g} / \mathrm{mL}$ ) for (a) $30 \mathrm{~min}$ and (b) $20 \mathrm{~min}$. These MAPK and MEK protein expressions were analyzed by western blot. Densitometric analysis was performed using ImageJ ver. 1.50i. The values are represented as the mean \pm S.D. $(n=3) .{ }^{\# \# \#} P<0.001$ vs. the CON group; ${ }^{* * *} P<0.001$ vs. the LPS-treated group.

released by cytochrome p450 metabolism, peroxisomes, mitochondria, and inflammatory cell activation by endotoxins in macrophages [35]. ROS have been associated with numerous proinflammatory signal transduction cascades triggered by IL- $1 \beta$, TNF- $\alpha$, and LPS [36]. It was reported that ROS have the latent ability to activate NF- $\kappa \mathrm{B}$ in the early disease phase [37]. Here, HO-1 is important for protecting macrophages from ROS and has become an interesting target for antioxidant-related experiments [12]. In addition, its products, such as iron ions, CO, and biliverdin, modulate inflammation and exhibit critical effects against oxidative stress [38].

Nrf2 signaling constitutes one of the crucial cellular defense mechanisms in xenobiotic damage and oxidative stress [13]. Activation of Keap1/Nrf2 signaling manifests advantageous effects in a several disease states including diabetes, atherosclerosis, malaria, liver injury, obesity, neurodegenerative diseases, and certain cancers [39]. Under normal physiological conditions, Nrf2 is sequestrated in the cytoplasm and, upon stimulation, isolated from its cytosolic inhibitor Keap1, translocated into the nucleus, and then combined to the cis-acting antioxidant responsible element
(ARE) in the promoter region [40]. The antioxidant features of ARE enzymes are involved in reducing the formation of adhesion molecules, such as NO and inhibition of oxidative stress [41]. It is also well known that there is crosstalk between NF- $\kappa$ B and Nrf2, which regulates proinflammatory responses. Moreover, overexpression of Nrf2 can suppress the activation of the NF- $\kappa \mathrm{B}$ pathway. Furthermore, the elimination of Nrf2 exacerbated the activation of NF- $\kappa \mathrm{B}$-induced inflammatory response [42]. Therefore, in this study, we examined whether CLME regulate the Keap1/Nrf2 signaling pathway, which is relevant to NO and ROS production, as well as the NF- $\kappa \mathrm{B}$ signaling. CLME effectively increased the nuclear accumulation of Nrf2 and concurrently downregulated the nuclear expression of NF- $\kappa \mathrm{B}$ in LPS-stimulated RAW 264.7 cells.

There are three well-defined subgroups of MAPKs in mammalian cells: ERK, JNK, and p38 MAPK. MAPKs are a chain of kinases connected with cell proliferation, cell survival, inflammation, apoptosis, tumorigenesis, and other physiological process [43]. Li et al. reported that the elevated ROS levels can activate a cascade of noxious events during inflammatory processes via MAPKs pathway, and ERK and 


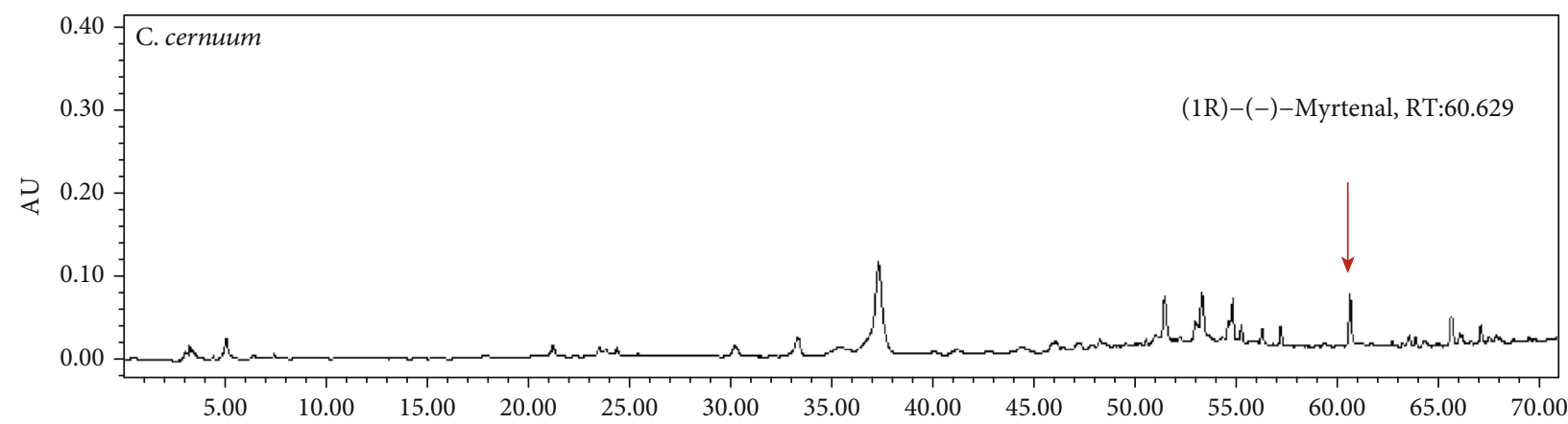

(a)

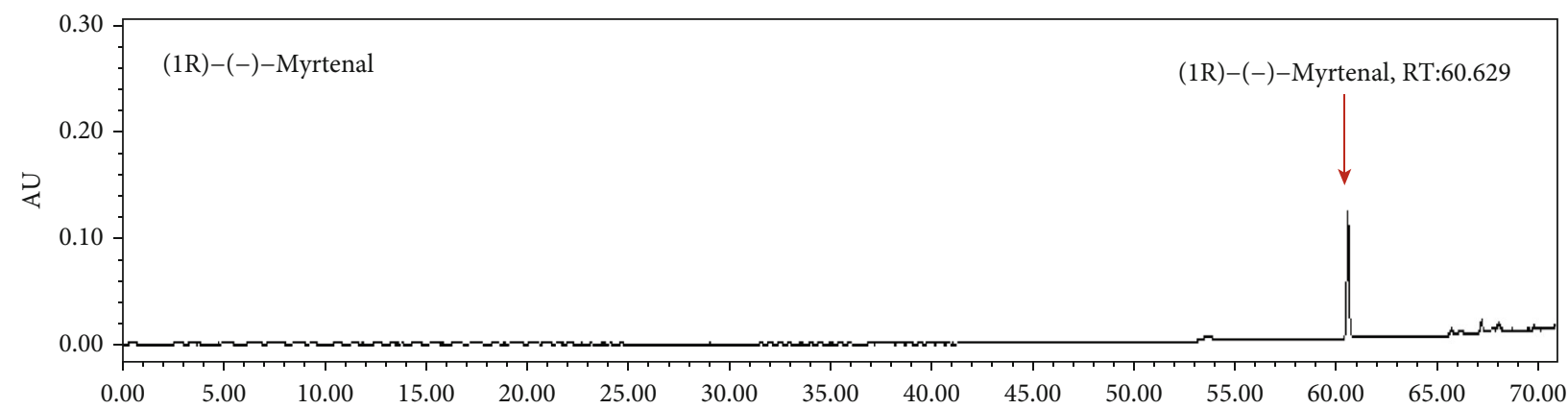

(b)

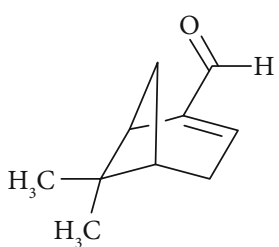

(c)

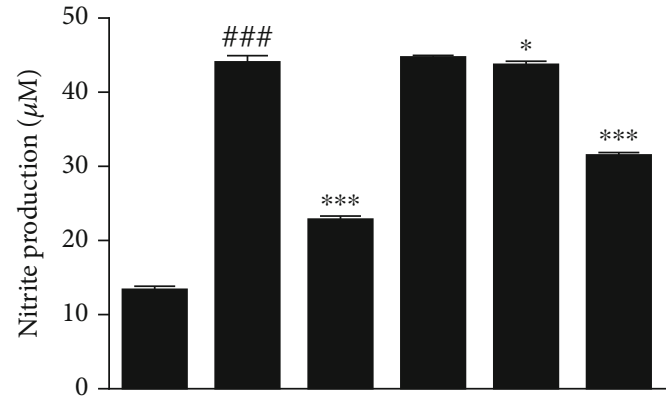

LPS $(\mu \mathrm{g} / \mathrm{mL})$

NIL $(\mu \mathrm{M})$

Myrtenal $(\mu \mathrm{M})$

- $\quad-$

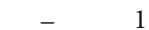

$-$

(e)

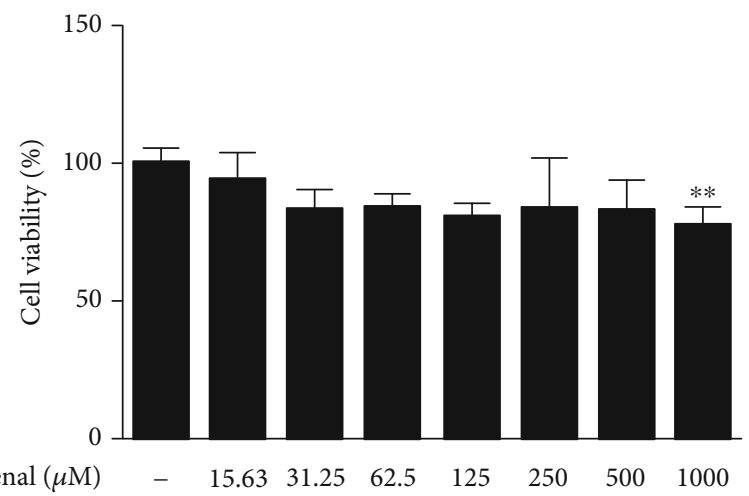

(d)

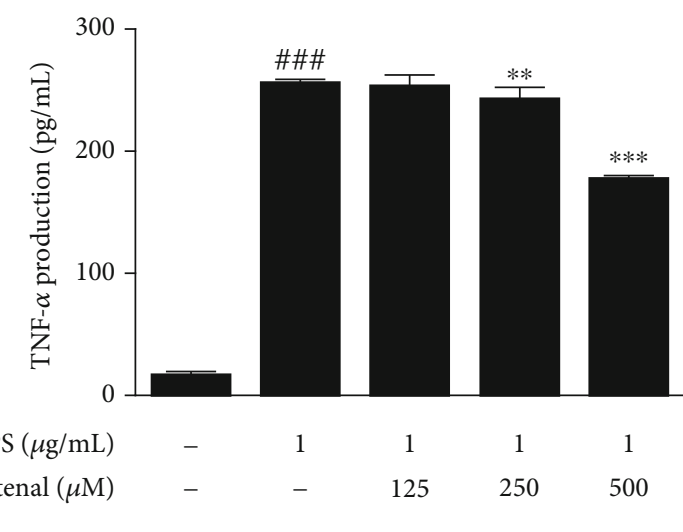

(f)

Figure 6: Continued 


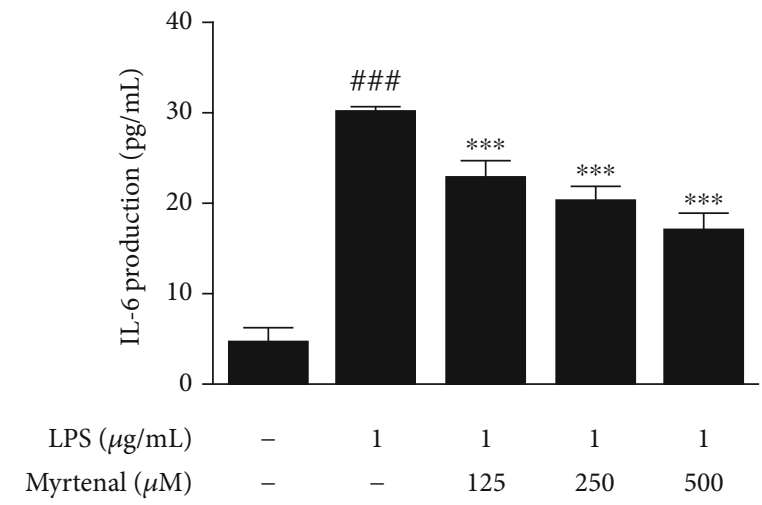

(g)

FIgURE 6: Myrtenal inhibits the production of NO, TNF- $\alpha$, and IL-6 in LPS-stimulated RAW 264.7 cells. HPLC chromatogram of CLME (a) and (1R)-(-)-Myrtenal (b) as a standard compound. (c) Chemical structures of Myrtenal. (d) The viability of Myrtenal in RAW 264.7 cells. The values are represented as the mean \pm S.D. $(n=6) .{ }^{* *} P<0.01$ vs. the CON group. (e) The nitrite production was determined using Griess reagent. (f) TNF- $\alpha$ and (g) IL- 6 production in the culture media was measured by ELISA kit. The values are represented as the mean \pm S.D. $(n=3)$. ${ }^{\# \#} P<0.001$ vs. the CON group; ${ }^{*} P<0.05,{ }^{* *} P<0.01$, and ${ }^{* * *} P<0.001$ vs. the LPS-treated group.

p38 MAPK were reported to be strongly implicated in Nrf2 pathways [44]. Additionally, the phosphorylation of MAPKs in LPS-challenged macrophages can also stimulate the transcriptional activation of NF- $\kappa$ B [29]. Our data demonstrated the phosphorylation of ERK, following LPS induction, was diminished by CLME treatment in the RAW 264.7 cells. Furthermore, CLME treatment also effectively alleviated the expression of $\mathrm{p}$-MEK, upstream of $\mathrm{p}$-ERK, compared to the LPS-treated group. The importance of ERK on inflammation, in particular, has been well reported. ERK is majorly correlated with LPS-induced macrophages inflammation [27, 45-48]. Additionally, it was reported that the suppression of ERK successfully inhibited inflammation in mouse and rabbit models $[49,50]$. Therefore, ERK inhibitors can be effective targets for therapeutic intervention in inflammatory diseases. Collectively, these data suggested that inactivation of MEK/ERK expression could be involved in the action of CLME on the expression of NF- $\kappa \mathrm{B}$ and Keap1/Nrf2 pathway.

Excessive inflammation is the main cause of organ failure and mortality in sepsis [51], which is induced by pathogens like LPS-releasing Gram-negative bacteria [52]. CLME administration enhanced the survival rate in mice upon sepsis induction but not reached significance, indicating that CLME may slightly protect the mice from the septic shock. Although further investigations of animal experiment are still needed to prove that CLME has an anti-inflammatory effect in vivo, the present results support that CLME may inhibit the LPS-stimulated inflammation at least in vitro.

Previously, the principal constituents of essential oil of C. cernuum were identified as sesquicineole, $\alpha$-bisabolol, and Myrtenal [19]. Among them, Myrtenal, a natural monoterpene, is one of the most abundant compounds in the Asteraceae family and is also a ubiquitous constituent of the essential oils of flowers, stems, and leaves [53]. In addition, Myrtenal is present in many medicinal plants such as pepper, cumin, mint, eucalyptus, and others [54]. Several studies have reported the various biological activities of Myrtenal, including antihepatocellular carcinoma activity in rats [55], antioxidant action against colon cancer in rats [56], and antihyperglycemic activity in diabetic rats [57]. Hari Babu et al. suggested that Myrtenal is being a good scavenger of $\mathrm{NO}$, as well as a free radical inhibitor [58]. In addition, Myrtenal-treated rats showed significant decreases in ROS levels compared to the 1,2-dimethyl hydrazine- (DMH-) induced colon cancer rat model [56]. Similarly, we found that Myrtenal is one of the major compounds in CLME, and it significantly suppressed NO, TNF- $\alpha$, and IL- 6 production in LPS-stimulated RAW 264.7 cells, showing that our result is consistent with previous studies. Myrtenal, an active compound of CLME, may be associated with powerful effects of CLME against inflammation and oxidative stress in LPS-stimulated RAW 264.7 cells.

However, our study has some limitations. First, our experiment design did not allow us to examine in more depth the major compounds of CLME, although our data support a role of Myrtenal for anti-inflammatory effects. Second, we set the concentrations of Myrtenal in vitro higher than the content of that in CLME identified by HPLC. So we are going to do a further study for other major compounds of CLME and assessing Myrtenal with content in CLME on inflammatory responses. The inhibitory effects of CLME in LPSstimulated RAW 264.7 cells could partially be involved in Myrtenal, which needs further investigation.

\section{Conclusions}

In our study, CLME revealed antioxidant/anti-inflammatory effects by regulating MEK/ERK, NF- $\kappa \mathrm{B}$, and Keap1/Nrf2 signaling in LPS-stimulated RAW 264.7 macrophages, and these effects may be correlated with Myrtenal, one of the major compounds in CLME.
Abbreviations
C. cernuum: Carpesium cernuum L
CLME: Methanolic extract of C. cernuum 


$\begin{array}{ll}\text { ERK: } & \text { Extracellular signal-related kinase } \\ \text { HO-1: } & \text { Heme oxygenese-1 } \\ \text { LPS: } & \text { Lipopolysaccharide } \\ \text { MAPK: } & \text { Mitogen-activating protein kinase } \\ \text { MEK: } & \text { ERK kinase } \\ \text { NF- } \kappa \text { B: } & \text { Nuclear translocation of nuclear factor- } \kappa \text { B } \\ \text { NO: } & \text { Nitric oxide } \\ \text { Nrf2: } & \text { Nuclear factor-erythroid } 2 \text { p45-related factor } 2 \\ \text { Keap1: } & \text { Kelch-like ECH-associated protein } 1 \\ \text { IL-6: } & \text { Interleukin-6 } \\ \text { TNF- } \alpha: & \text { Tumor necrosis factor- } \alpha \\ \text { PGE }: & \text { Prostaglandin } \mathrm{E}_{2} \\ \text { ROS: } & \text { Reactive oxygen species. }\end{array}$

\section{Data Availability}

The datasets used and/or analyzed in this study are available from the corresponding authors on reasonable request.

\section{Disclosure}

This study was composed of a master's thesis of Yea-Jin Park.

\section{Conflicts of Interest}

The authors declare that there is no conflict of interest regarding the publication of this paper.

\section{Authors' Contributions}

Y.J.P. and H.J.A. conceived and designed the experiments. Y.J.P. acquired the data and performed the experiments and wrote the manuscript. S.Y.C. revised the data and provided technical support. D.S.L. contributed the reagents, materials, and analysis tools about HPLC experiments. D.C.C. conducted the animal experiments. Z.Z. and S.L. provided the reagents and materials related to medicinal plant. H.J.A. substantially contributed to the analysis and interpretation of data and revised the manuscript. All authors read and approved the final manuscript.

\section{Acknowledgments}

This research was supported by the Basic Science Program through the National Research Foundation of Korea (NRF), funded by the Ministry of Science, ICT \& Future Planning [NRF-2019R1A2C4070234], and the KRIBB Initiative Program of the Republic of Korea.

\section{Supplementary Materials}

Table 1: the time point of all markers. Fig. S1: effect of CLME on the viability of RAW 264.7 cells. Fig. S2: CLME increases the expression of HO-1 in LPS-stimulated RAW 264.7 cells. (Supplementary Materials)

\section{References}

[1] D. M. Pereira, G. Correia-da-Silva, P. Valentão, N. Teixeira, and P. B. Andrade, "Anti-inflammatory effect of unsaturated fatty acids and Ergosta-7,22-dien-3-ol from Marthasterias glacialis: prevention of CHOP-mediated ER-stress and NF- $\kappa \mathrm{B}$ activation," PLoS One, vol. 9, no. 2, article e88341, 2014.

[2] P. Wang, Q. Qiao, J. Li, W. Wang, L. P. Yao, and Y. J. Fu, "Inhibitory effects of geraniin on LPS-induced inflammation via regulating NF- $\kappa$ B and Nrf2 pathways in RAW 264.7 cells," Chemico-Biological Interactions, vol. 253, pp. 134-142, 2016.

[3] S. Mohan and D. Gupta, "Crosstalk of toll-like receptors signaling and Nrf2 pathway for regulation of inflammation," Biomedicine \& Pharmacotherapy, vol. 108, pp. 1866-1878, 2018.

[4] H. J. Lee, J. S. Shin, K. G. Lee et al., "Ethanol extract of Potentilla supina Linne suppresses LPS-induced inflammatory responses through NF- $\kappa \mathrm{B}$ and $\mathrm{AP}-1$ inactivation in macrophages and in endotoxic mice," Phytotherapy Research, vol. 31, no. 3, pp. 475-487, 2017.

[5] Y.-C. Oh, W.-K. Cho, J. H. Oh et al., "Fermentation by Lactobacillus enhances anti-inflammatory effect of Oyaksungisan on LPS-stimulated RAW 264.7 mouse macrophage cells," BMC Complementary and Alternative Medicine, vol. 12, 2012.

[6] T. K. Lee, E. M. Denny, J. C. Sanghvi et al., "A noisy paracrine signal determines the cellular NF-kappaB response to lipopolysaccharide," Science Signaling, vol. 2, no. 93, 2009.

[7] Y. H. Choi, G.-Y. Kim, and H. H. Lee, "Anti-inflammatory effects of cordycepin in lipopolysaccharide-stimulated RAW 264.7 macrophages through Toll-like receptor 4-mediated suppression of mitogen-activated protein kinases and NF- $\kappa \mathrm{B}$ signaling pathways," Drug Design, Development and Therapy, vol. 8, pp. 1941-1953, 2014.

[8] K. Muniandy, S. Gothai, K. M. H. Badran, S. S. Kumar, N. M. Esa, and P. Arulselvan, "Suppression of Proinflammatory Cytokines and Mediators in LPS-Induced RAW 264.7 Macrophages by Stem Extract of Alternanthera sessilis via the Inhibition of the NF- $\kappa$ B Pathway," Journal of Immunology Research, vol. 2018, Article ID 3430684, 12 pages, 2018.

[9] J. S. Shin, H. T. Im, and K. T. Lee, "Saikosaponin B2 Suppresses Inflammatory Responses Through IKK/I $\kappa \mathrm{B} \alpha / \mathrm{NF}-\kappa \mathrm{B}$ Signaling Inactivation in LPS-Induced RAW 264.7 Macrophages," Inflammation, vol. 42, pp. 342-353, 2019.

[10] C. Yang, C. Zhang, Z. Wang, Z. Tang, H. Kuang, and A.N. Kong, "Corynoline isolated from Corydalis bungeana Turcz. exhibits anti-inflammatory effects via modulation of Nfr2 and MAPKs," Molecules, vol. 21, no. 8, 2016.

[11] N. Khurana and S. C. Sikka, "Targeting crosstalk between Nrf2, NF-kappaB and androgen receptor signaling in prostate cancer," Cancers, vol. 10, no. 10, 2018.

[12] X. Xu, H. Li, X. Hou et al., "Punicalagin Induces Nrf2/HO-1 Expression via Upregulation of PI3K/AKT Pathway and Inhibits LPS-Induced Oxidative Stress in RAW264.7 Macrophages," Mediators of Inflammation, vol. 2015, Article ID 380218, 11 pages, 2015.

[13] Y. S. Chiou, Q. Huang, C. T. Ho, Y. J. Wang, and M. H. Pan, "Directly interact with Keap1 and LPS is involved in the anti-inflammatory mechanisms of (-)-epicatechin-3-gallate in LPS-induced macrophages and endotoxemia," Free Radical Biology \& Medicine, vol. 94, pp. 1-16, 2016.

[14] C. Guo, L. Yang, J. Luo et al., "Sophoraflavanone G from Sophora alopecuroides inhibits lipopolysaccharide-induced inflammation in RAW264.7 cells by targeting PI3K/Akt, JAK/STAT and Nrf2/HO-1 pathways," International Immunopharmacology, vol. 38, pp. 349-356, 2016. 
[15] C. H. Jin, Y. K. So, S. N. Han, and J. B. Kim, "Isoegomaketone upregulates heme oxygenase-1 in RAW264.7 cells via ROS/p38 MAPK/Nrf2 pathway," Biomolecules \& Therapeutics, vol. 24, no. 5, pp. 510-516, 2016.

[16] S. Khatua and K. Acharya, "Water soluble antioxidative crude polysaccharide from Russula senecis elicits TLR modulated $\mathrm{NF}-\kappa \mathrm{B}$ signaling pathway and pro-inflammatory response in murine macrophages," Frontiers in Pharmacology, vol. 9, 2018.

[17] Z. Wu, T. Zhou, and P. Xiao, "XinHuaBenCaoGangYao.," List of Chinese Medicine Herb, vol. 1, pp. 398-399, 1990.

[18] http://www.zysj.com.cn/.

[19] L. S. Bisht, R. Prasad, D. Joshi, M. K. Nailwal, and A. B. Melkani, "Phytochemical screening and antibacterial assay of the essential oil from Carpesium cernuum L," International Journal of Green and Herbal Chemistry, vol. 6, no. 4, pp. 107116, 2017.

[20] H. Wu, H. Pang, Y. Chen et al., "Anti-Inflammatory Effect of a Polyphenol-Enriched Fraction from Acalypha wilkesiana on Lipopolysaccharide-Stimulated RAW 264.7 Macrophages and Acetaminophen-Induced Liver Injury in Mice," Oxidative Medicine and Cellular Longevity, vol. 2018, Article ID 7858094, 17 pages, 2018.

[21] S. De, A. Manna, S. Kundu et al., "Allylpyrocatechol attenuates collagen-induced arthritis via attenuation of oxidative stress secondary to modulation of the MAPK, JAK/STAT, and Nrf2/HO-1 pathways," The Journal of Pharmacology and Experimental Therapeutics, vol. 360, no. 2, pp. 249-259, 2017.

[22] Y. Cheng, C. Yang, D. Luo, X. Li, X. C. le, and J. Rong, "N-Propargyl caffeamide skews macrophages towards a resolving M2like phenotype against myocardial ischemic injury via activating Nrf2/HO-1 pathway and inhibiting NF-kB pathway," Cellular Physiology and Biochemistry, vol. 47, no. 6, pp. 25442557, 2018.

[23] Y. Sugiyama, Y. Hiraiwa, Y. Hagiya, M. Nakajima, T. Tanaka, and S. I. Ogura, "5-Aminolevulinic acid regulates the immune response in LPS-stimulated RAW 264.7 macrophages," BMC Immunology, vol. 19, no. 1, 2018.

[24] L. Merly and S. L. Smith, "Murine RAW 264.7 cell line as an immune target: are we missing something?," Immunopharmacology and Immunotoxicology, vol. 39, no. 2, pp. 55-58, 2017.

[25] R. P. Kumar and A. Abraham, "Inhibition of LPS induced proinflammatory responses in RAW 264.7 macrophage cells by PVP-coated naringenin nanoparticle via down regulation of NF- $\kappa$ B/P38MAPK mediated stress signaling," Pharmacological Reports, vol. 69, no. 5, pp. 908-915, 2017.

[26] J. Park, C. H. Kwak, S. H. Ha et al., "Ganglioside GM3 suppresses lipopolysaccharide-induced inflammatory responses in rAW 264.7 macrophage cells through NF- $\kappa$ B, AP-1, and MAPKs signaling," Journal of Cellular Biochemistry, vol. 119, no. 1, pp. 1173-1182, 2018.

[27] H. Sun, W. Cai, X. Wang et al., "Vaccaria hypaphorine alleviates lipopolysaccharide-induced inflammation via inactivation of NF $\kappa$ B and ERK pathways in Raw 264.7 cells," BMC Complementary and Alternative Medicine, vol. 17, no. 1, 2017.

[28] K. S. Robbins, "Effect of pecan phenolics on the release of nitric oxide from murine RAW 264.7 macrophage cells," Food Chemistry, vol. 212, pp. 681-687, 2016.

[29] H. Harikrishnan, I. Jantan, M. A. Haque, and E. Kumolosasi, "Anti-inflammatory effects of Phyllanthus amarus Schum. \& Thonn. through inhibition of NF- $\kappa$ B, MAPK, and PI3K-Akt signaling pathways in LPS-induced human macrophages,"
BMC Complementary and Alternative Medicine, vol. 18, no. 1,2018

[30] P. Viatour, M. P. Merville, V. Bours, and A. Chariot, "Phosphorylation of NF-kappaB and IkappaB proteins: implications in cancer and inflammation," Trends in Biochemical Sciences, vol. 30, no. 1, pp. 43-52, 2005.

[31] M. Lee, N. Y. Lee, K.-S. Chung, S.-Y. Cheon, K.-T. Lee, and H.J. An, "Roxatidine attenuates mast cell-mediated allergic inflammation via inhibition of NF- $\kappa$ B and p38 MAPK activation," Scientific Reports, vol. 7, no. 1, article 41721, 2017.

[32] W. Guo, J. Sun, L. Jiang et al., "Imperatorin attenuates LPSinduced inflammation by suppressing NF- $\kappa \mathrm{B}$ and MAPKs activation in RAW 264.7 macrophages," Inflammation, vol. 35, no. 6, pp. 1764-1772, 2012.

[33] N. Khansari, Y. Shakiba, and M. Mahmoudi, "Chronic inflammation and oxidative stress as a major cause of age-related diseases and cancer," Recent Patents on Inflammation \& Allergy Drug Discovery, vol. 3, no. 1, pp. 73-80, 2009.

[34] S. Chatterjee, "Oxidative stress, inflammation, and disease," in Oxidative stress and biomaterials, pp. 35-58, Elsevier, 2016.

[35] H. L. Yang, S. W. Lin, C. C. Lee et al., "Induction of Nrf2mediated genes by Antrodia salmonea inhibits ROS generation and inflammatory effects in lipopolysaccharide-stimulated RAW264.7 macrophages," Food \& Function, vol. 6, no. 1, pp. 230-241, 2015.

[36] Q. Li and J. F. Engelhardt, "Interleukin-1 $\beta$ Induction of NF $\kappa \mathrm{B}$ Is Partially Regulated by $\mathrm{H}_{2} \mathrm{O}_{2}$-mediated Activation of $\mathrm{NF} \kappa \mathrm{B}$ inducing Kinase," The Journal of Biological Chemistry, vol. 281, no. 3, pp. 1495-1505, 2006.

[37] S. Nakajima and M. Kitamura, "Bidirectional regulation of NF- $\kappa \mathrm{B}$ by reactive oxygen species: a role of unfolded protein response," Free Radical Biology \& Medicine, vol. 65, pp. 162 174, 2013.

[38] T. U. Jayawardena, K. K. Asanka Sanjeewa, I. P. Shanura Fernando et al., "Sargassum horneri (Turner) C. Agardh ethanol extract inhibits the fine dust inflammation response via activating Nrf2/HO-1 signaling in RAW 264.7 cells," BMC Complementary and Alternative Medicine, vol. 18, no. 1, 2018.

[39] N. K. H. Khoo, L. Li, S. R. Salvatore, F. J. Schopfer, and B. A. Freeman, "Electrophilic fatty acid nitroalkenes regulate Nrf2 and NF- $\kappa$ B signaling:a medicinal chemistry investigation of structure-function relationships," Scientific Reports, vol. 8, no. 1, article 2295, 2018.

[40] K. K. J. Senthil, V. M. Gokila, J.-L. Mau et al., "A steroid like phytochemical Antcin $\mathrm{M}$ is an anti-aging reagent that eliminates hyperglycemia-accelerated premature senescence in dermal fibroblasts by direct activation of Nrf2 and SIRT-1," Oncotarget, vol. 7, no. 39, pp. 62836-62861, 2016.

[41] M. Gasparrini, T. Y. Forbes-Hernandez, F. Giampieri et al., "Anti-inflammatory effect of strawberry extract against LPSinduced stress in RAW 264.7 macrophages," Food and Chemical Toxicology, vol. 102, pp. 1-10, 2017.

[42] K.-W. Kim, C.-S. Yoon, Y.-C. Kim, and H. Oh, "Desoxonarchinol A and narchinol B isolated from Nardostachys jatamansi exert anti-neuroinflammatory effects by up-regulating of nuclear transcription factor erythroid-2-related factor 2/heme oxygenase-1 signaling," Neurotoxicity Research, vol. 35, pp. 230-243, 2019.

[43] L. Cao, Y. Xue, Z. Yang et al., "Shikonofuran E plays an antiinflammatory role by down-regulating MAPK and NFkappaB signaling pathways in lipopolysaccharide-stimulated 
RAW264.7 macrophages," Journal of Natural Medicines, vol. 73, pp. 244-251, 2019.

[44] C. Li, T. Chen, H. Zhou et al., "Schisantherin A attenuates neuroinflammation in activated microglia: role of Nrf2 activation through ERK phosphorylation," Cellular Physiology and Biochemistry, vol. 47, no. 5, pp. 1769-1784, 2018.

[45] G. Xu, L. Feng, P. Song et al., "Isomeranzin suppresses inflammation by inhibiting M1 macrophage polarization through the NF- $\kappa \mathrm{B}$ and ERK pathway," International Immunopharmacology, vol. 38, pp. 175-185, 2016.

[46] J. Liu, J. Tang, Y. Zuo et al., "Stauntoside B inhibits macrophage activation by inhibiting NF- $\kappa$ B and ERK MAPK signalling," Pharmacological Research, vol. 111, pp. 303-315, 2016.

[47] H. Lv, H. Ren, L. Wang, W. Chen, and X. Ci, "Lico A Enhances Nrf2-Mediated Defense Mechanisms against $t$-BHP-Induced Oxidative Stress and Cell Death via Akt and ERK Activation in RAW 264.7 Cells," Oxidative Medicine and Cellular Longevity, vol. 2015, Article ID 709845, 13 pages, 2015.

[48] Y. K. Choi, B. R. Ye, E. A. kim et al., "Bis (3-bromo-4,5-dihydroxybenzyl) ether, a novel bromophenol from the marine red alga Polysiphonia morrowii that suppresses LPS-induced inflammatory response by inhibiting ROS-mediated ERK signaling pathway in RAW 264.7 macrophages," Biomedicine \& Pharmacotherapy, vol. 103, pp. 1170-1177, 2018.

[49] B. D. Jaffee, E. J. Manos, R. J. Collins et al., "Inhibition of MAP Kinase Kinase (MEK) Results in an Anti-inflammatory Response in Vivo," Biochemical and Biophysical Research Communications, vol. 268, no. 2, pp. 647-651, 2000.

[50] J. P. Pelletier, J. C. Fernandes, J. Brunet et al., "In vivo selective inhibition of mitogen-activated protein kinase kinase $1 / 2$ in rabbit experimental osteoarthritis is associated with a reduction in the development of structural changes," Arthritis and Rheumatism, vol. 48, no. 6, pp. 1582-1593, 2003.

[51] G. He, X. Zhang, Y. Chen, J. Chen, L. Li, and Y. Xie, "Isoalantolactone inhibits LPS-induced inflammation via NF- $\kappa$ B inactivation in peritoneal macrophages and improves survival in sepsis," Biomedicine \& Pharmacotherapy, vol. 90, pp. 598607, 2017.

[52] H. Wu, J. Liu, W. Li, G. Liu, and Z. Li, "LncRNA-HOTAIR promotes TNF- $\alpha$ production in cardiomyocytes of LPSinduced sepsis mice by activating NF- $\kappa$ B pathway," Biochemical and Biophysical Research Communications, vol. 471, no. 1, pp. 240-246, 2016.

[53] L. K. Booupathy, S. Venkatachalam, N. Natarajan, R. Thamaraiselvan, M. Arumugam, and B. Maruthaiveeran Periyasamy, "Chemopreventive effect of myrtenal on bacterial enzyme activity and the development of 1,2-dimethyl hydrazine-induced aberrant crypt foci in Wistar rats," Journal of Food and Drug Analysis, vol. 24, no. 1, pp. 206-213, 2016.

[54] L. H. Babu, S. Perumal, and M. P. Balasubramanian, "Myrtenal, a natural monoterpene, down-regulates TNF- $\alpha$ expression and suppresses carcinogen-induced hepatocellular carcinoma in rats," Molecular and Cellular Biochemistry, vol. 369, no. 12, pp. 183-193, 2012.

[55] H. B. Lingaiah, N. Natarajan, R. Thamaraiselvan, P. Srinivasan, and B. M. Periyasamy, "Myrtenal ameliorates diethylnitrosamine-induced hepatocarcinogenesis through the activation of tumor suppressor protein p53 and regulation of lysosomal and mitochondrial enzymes," Fundamental \& Clinical Pharmacology, vol. 27, no. 4, pp. 443-454, 2013.

[56] B. Lokeshkumar, V. Sathishkumar, N. Nandakumar, T. Rengarajan, A. Madankumar, and M. P. Balasubramanian,
"Anti-oxidative effect of myrtenal in prevention and treatment of colon cancer induced by 1,2-dimethyl hydrazine (DMH) in experimental animals," Biomolecules \& Therapeutics, vol. 23, no. 5, pp. 471-478, 2015.

[57] A. Rathinam and L. Pari, "Myrtenal ameliorates hyperglycemia by enhancing GLUT2 through Akt in the skeletal muscle and liver of diabetic rats," Chemico-Biological Interactions, vol. 256, pp. 161-166, 2016.

[58] L. H. Babu, S. Perumal, and M. P. Balasubramanian, "Myrtenal attenuates diethylnitrosamine-induced hepatocellular carcinoma in rats by stabilizing intrinsic antioxidants and modulating apoptotic and anti-apoptotic cascades," Cellular Oncology, vol. 35, no. 4, pp. 269-283, 2012. 